\title{
Reduction in case fatality rate from meningococcal disease associated with improved healthcare delivery
}

R Booy, P Habibi, S Nadel, C de Munter, J Britto, A Morrison, M Levin, and the Meningococcal Research Group

\begin{abstract}
Background and aims-The case fatality rate from meningococcal disease (MD) has remained relatively unchanged in the post antibiotic era, with $20-50 \%$ of patients who develop shock still dying. In 1992 a new paediatric intensive care unit (PICU) specialising in MD was opened. Educational information was disseminated to local hospitals, and a specialist transport service was established which delivered mobile intensive care. The influence of these changes on mortality of children with MD was investigated.
\end{abstract}

Methods-A total of 331 consecutive children with meningococcal disease admitted to the PICU between 1992 and 1997 were studied. Severity of the disease on admission was assessed using the paediatric risk of mortality (PRISM) score. Logistic regression analysis was used to correct for clinical severity, age, and sex; death was the outcome, and year of admission, a temporal trend variable, was the primary exposure.

Results-The case fatality rate fell year on year (from $23 \%$ in $1992 / 93$ to $2 \%$ in 1997) despite disease severity remaining largely unchanged. After adjustment for age, sex, and disease severity, the overall estimate for improvement in the odds of death was $59 \%$ per year (odds ratio for the yearly trend 0.41).

Conclusions-A significant improvement in outcome for children admitted with $M D$ to a PICU has occurred in association with improvements in initial management of patients with MD at referring hospitals, use of a mobile intensive care service, and centralisation of care in a specialist unit. (Arch Dis Child 2001;85:386-390)

Keywords: meningococcal disease; survival; intensive care

Meningococcal disease (MD) remains an important cause of mortality and morbidity for children and young adults in both industrialised and developing countries. ${ }^{12}$ The published literature indicates that mortality from $\mathrm{MD}$ has not changed significantly since the introduction of antibiotics ${ }^{3} ; 10 \%$ of patients overall, and $20-50 \%$ of those who develop meningococcal shock do not survive the disease.$^{5-8}$ Survivors may suffer permanent disability as a result of amputation of limbs or digits, extensive scarring, or neurological injury. $^{8}$

A number of scoring systems have been developed to identify severely affected patients in need of intensive care. ${ }^{79^{10}}$ More recently, clinical severity scoring systems have been used to select patients, predicted to be at high risk of death, for inclusion in studies of experimental treatments such as extracorporeal membrane oxygenation (ECMO), ${ }^{11}$ haemofiltration, ${ }^{12}$ or thrombolytic, ${ }^{13}$ anticoagulant, ${ }^{14}$ or antiendotoxin therapies. ${ }^{15}$ Benefits for a number of experimental treatments have been claimed based on an apparent improvement in outcome when compared with either historical controls, or with death rates predicted by scoring systems. ${ }^{11-15}$

Apart from the use of the above experimental approaches, there have, over the past few years, been major changes in the routine management of patients with $\mathrm{MD}$, as a result of increased early use of parenteral penicillin by general practitioners (GPs), improved resuscitation in local hospitals, development of mobile intensive care, centralisation of care in specialist paediatric intensive care units, and improvements in the management of shock, acute lung dysfunction, and multiorgan failure. ${ }^{16}$

In order to investigate whether change in management has resulted in improved outcome, and to define the current case fatality rate for patients receiving paediatric intensive care, we have studied 331 consecutive patients with meningococcal disease admitted to a specialist paediatric intensive care unit (PICU) at St Mary's Hospital, London, over a 5 year 7 month period beginning June 1992. The risk of death over time was modelled by logistic regression controlling for severity of disease. The analysis shows that there has been a substantial, progressive improvement in outcome over this period.

\section{Patients and methods}

CASE DEFINITIONS

In children admitted to the PICU at St Mary's Hospital between June 1992 and December 1997, the diagnosis of MD was confirmed by isolation of $N$ meningitidis from blood, CSF, or the nasopharynx, the detection by latex agglutination of meningococcal antigens in CSF or blood, or detection of meningococcal DNA by polymerase chain reaction. Patients in whom diagnostic tests were negative but with characteristic features of $\mathrm{MD}$ were included if no 
alternative bacterial or viral cause for the illness was identified. The clinical diagnosis of MD required the presence of fever and haemorrhagic rash together with clinical features of meningitis (and/or raised intracranial pressure) or septicaemia with either hypotension or two or more features of end organ underperfusion (raised base deficit ( $>8 \mathrm{mmol}$ ), oliguria despite adequate volume replacement $(<1 \mathrm{ml} / \mathrm{kg} / \mathrm{h})$, temperature gradient $>3^{\circ} \mathrm{C}$ between the central and peripheral temperatures, declining neurological function, or a $\mathrm{PaO}_{2} / \mathrm{FiO}_{2}$ ratio of $<26.6 \mathrm{kPa}$ ). The case fatality rate was defined as the proportion of MD patients admitted each year that died within one month of admission.

DISEASE SEVERITY

Severity of MD was assessed using a severity of illness score, the Paediatric Risk of Mortality (PRISM) score, ${ }^{9}$ which has been shown to be predictive of outcome of meningococcal disease. ${ }^{10}$ The PRISM score's calculation is based on data collected over a 24 hour period. We timed the measurement of PRISM from when the PICU was contacted (rather than when the patient arrived in the PICU) because from that time, advice on intensive care management was given and acted on; furthermore, this "pretransport" PRISM has been accepted as an accurate predictor of the hospital mortality rate. ${ }^{17}$ Trained staff determined all scores and each score was independently calculated by at least two health professionals; any discrepancies were then discussed and resolved. The aim of using an objective score to grade disease severity was to enable an analysis of the risk of death which is not confounded by a change in the severity of MD over time; without a measure of clinical severity, a fall in the proportion of MD cases that die may merely reflect the admission of less severely affected patients.

\section{CLINICAL MANAGEMENT}

Few illnesses are as rapidly progressive as meningococcal sepsis. When the diagnosis is suspected, GPs are advised to promptly administer parenteral penicillin ${ }^{18}$ and arrange immediate transfer to the local hospital. Until recently, severely affected patients were often considered too ill to be transferred to PICUs in regional centres, and therefore they were usually treated either in paediatric wards or adult intensive care units at district general hospitals. In June 1992 a new specialist meningococcal research unit and PICU was established at St Mary's Hospital, London, with the specific aims of centralising the care of children with meningococcal sepsis and undertaking research to improve the management of the disorder. Paediatric departments throughout the south of England were informed of the new unit, and encouraged to contact the PICU as soon as possible after admission of a child with MD so as to discuss referral of patients who were severely affected for treatment and inclusion in clinical trials. Information on the management of MD was disseminated to clinicians in district general hospital paediatric departments, accident and emergency departments, and adult intensive care units, through a series of seminars and meetings. Additionally published guidelines on the management of MD were also mailed to consultant paediatricians and the recommended management was updated as new information emerged. ${ }^{16} 19$

A mobile intensive care team led by a senior paediatric intensivist, and a senior paediatric intensive care nurse transferred all children referred to the PICU. Telephone advice regarding emergency resuscitation and stabilisation was provided by the attending consultant intensivist prior to the arrival of the mobile intensive care team at the local hospital. A dialogue continued through repeated telephone calls while the team was en route. The concept of mobile intensive care was developed to enable a similar level of care to that available in the PICU to be delivered at local hospitals. Elective tracheal intubation and ventilation (if not already done), haemodynamic monitoring, and ongoing resuscitation, were undertaken by the mobile intensive care team at the local hospital. Patients were stabilised prior to transfer back to the PICU. Photocopies of local hospital notes and GP referral letters were taken for inclusion in the PICU notes. Whether patients received parenteral penicillin prior to referral to hospital was routinely recorded.

Intensive care management has been fully described elsewhere. ${ }^{16}{ }^{19} 20$ It included aggressive resuscitation of the circulation by plasma volume expansion (preferably with $4.5 \%$ albumin), early elective tracheal intubation and ventilation, use of inotropes, correction of coagulopathy, correction of metabolic derangements (for example, potassium, calcium, magnesium, phosphate, bicarbonate, and glucose) and early renal replacement therapy. For patients requiring renal replacement, either peritoneal dialysis or continuous veno-venous haemofiltration with or without dialysis were performed.

Patients who survived were re-evaluated in the outpatient department six to eight weeks after the acute illness. The frequency of amputation of limbs or digits, the requirement for skin grafting, and extent of neurological injury (for example, hemiparesis, sensorineural deafness, seizure disorder) were assessed.

\section{STATISTICAL ANALYSES}

The statistical programs Stata (version 5) and SAS were used. The trend in case fatality rate was examined over a 5 year 7 month period beginning June 1992. Logistic regression analysis was performed to model the temporal trend in risk of death. Year of admission to PICU was the main explanatory variable. As only seven patients were admitted in 1992, data for this year were combined with 1993. Adjustment for various covariates was made. These included age, sex, and clinical severity (as measured by PRISM). The contribution of GP administered parenteral penicillin was examined in the model after exclusion of the clinical severity score. Modelling was done using a forward selection procedure. The analysis was repeated after inclusion of the patients who died before transfer to the PICU was possible. 


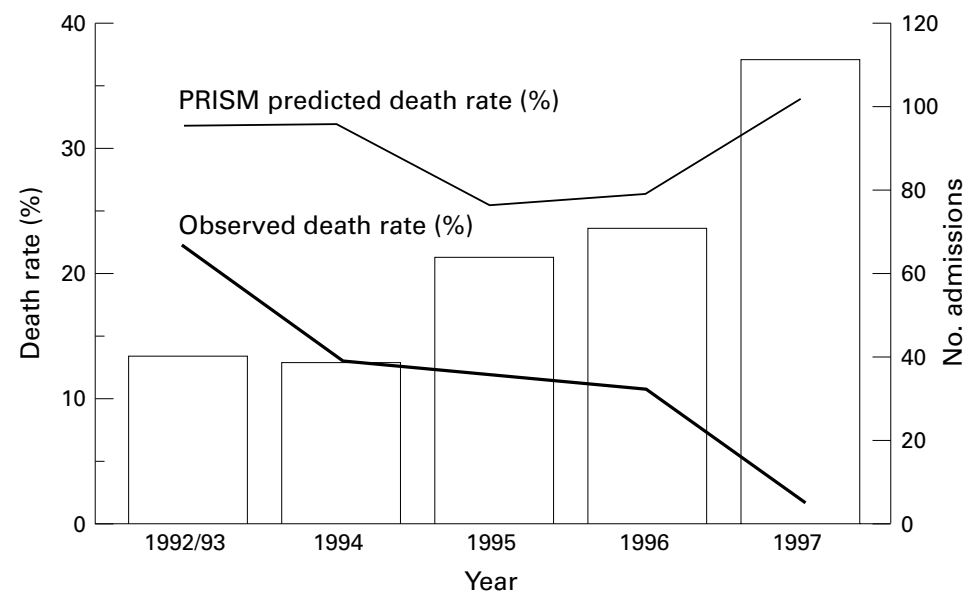

Figure 1 Number of yearly admissions of children with MD to the PICU at St Mary's Hospital, shown by the bars. Annual case fatality rates predicted by PRISM and actual annual case fatality rates are superimposed as line graphs.

The likelihood ratio test was employed to examine if the trend in case fatality rate was linear.

Between June 1992 and May 1995, 49 patients were enrolled at St Mary's PICU as part of a double blind, randomised, placebo controlled multicentre trial of a monoclonal antiendotoxin antibody, HA1A. Overall, this trial did not show a significant improvement in survival with HA1A. ${ }^{21}$ During 1997, 60 patients referred to PICU with severe MD were enrolled in a phase III double blind, randomised placebo controlled multicentre trial of recombinant human bactericidal/permeability increasing protein (rBPI), which has been shown to be safe and possibly beneficial in a phase I/II trial. ${ }^{15}$ Although the use of rBPI was subsequently found to significantly improve functional outcome, there was only a nonsignificant trend towards reduced mortality in the trial as a whole. ${ }^{22}$ To assess whether either of these two treatments had an effect on survival in children enrolled to St Mary's PICU, unblinded allocation data were used in a logistic regression model. This controlled for disease severity using the PRISM score. The likelihood ratio test was used and adjustment was made for the covariates found to contribute to the model that was fitted to the full data set.

\section{Results}

There were 331 children with MD admitted to the PICU between June 1992 and December 1997. Their median age was 2 years 8 months and ranged from 5 weeks to 17 years 8 months. Females accounted for 188 cases. Septicaemia was the principal diagnosis in 281 cases and meningitis in 50. The number of patients admitted in each year of the study increased rapidly following opening of the PICU in 1992 (fig 1). This was mainly a reflection of a greater proportion of the severe cases in the south of England being referred to the new unit, rather than an increase in the incidence of the disease, as over the same time period the numbers of cases within England and Wales increased much more slowly. ${ }^{23}$
Outcome was determined for all 331 patients. There were 33 deaths in the PICU (two in 1992, eight in 1993, five in 1994, eight in 1995, eight in 1996, and two in 1997), 29 with a principal diagnosis of septicaemia and four with meningitis. A further 29 children were referred but died before they could be brought back to the PICU (none in 1992, three in 1993, eight in 1994, ten in 1995, three in 1996, and five in 1997). The case fatality rate among PICU admissions in $1992 / 93$ was $23 \%$, close to the proportion predicted by the PRISM score (see fig 1) and consistent with previously reported death rates in children with severe meningococcal sepsis. ${ }^{6-8}$ However, thereafter the observed case fatality rate was progressively less than predicted by the PRISM score; the proportion of fatal outcomes fell year on year so that by 1997 only two of 111 MD patients admitted to the unit died, whereas PRISM predicted 38 deaths.

Logistic regression analysis, controlling for disease severity, age, and sex, showed that over the study period (1992-97) the overall estimate for the reduction in the odds of death was 59\% per year (odds ratio for the yearly trend 0.41 , $95 \%$ CI 0.27 to $0.62, p=0.000001)$. This estimate and its significance remained the same after inclusion of the 29 deaths that occurred at local hospitals. Using the likelihood ratio test to determine whether the relation between year of admission and outcome was linear, no significant improvement to the model was achieved by considering each year as a separate exposure strata.

Examination of fig 1 suggests that the degree of change in the observed death rate compared with the expected death rate was greater from 1996 to 1997 (a time period when the experimental therapy, rBPI, was undergoing trial) than in the intervening period of the study. However, unblinding of allocation revealed that the case fatality rate was higher in recipients of active treatment; regression analysis showed no significant effect of rBPI on survival $(p=0.41)$.

After excluding the PRISM score from the overall model, the addition of prehospital penicillin to the model did not confound the estimate of the effect of year of admission.

The proportion of survivors with complications changed little during the study period. The percentage of survivors requiring amputation or skin grafting was 5.3\% in 1992-95 and $5.8 \%$ in $1996-97$; the proportions with neurological abnormality were $9.7 \%$ in $1992-95$ and $7.3 \%$ in $1996-97$.

\section{Discussion}

Since the opening at St Mary's Hospital of a specialist PICU focused on research and treatment of $\mathrm{MD}$, there has been a substantial progressive reduction in the annual death rate. The case fatality rate currently observed is dramatically lower than that previously reported in the literature for children with severe $\mathrm{MD}$ requiring intensive care treatment. ${ }^{36-8}$ Given that the overall severity of cases admitted to PICU changed little over the course of the study and that the analyses involved controlling 
for potential confounders (age, sex, severity of disease on admission to hospital, and use of prehospital parenteral penicillin), the data suggest that a major reduction in mortality from the disease has been achieved.

The validity of this conclusion is dependent firstly on the accuracy of measurement of disease severity on admission to hospital and secondly, on the assumption that the confounding influence of disease severity has been adequately captured by a severity score. Misclassification may have occurred in MD severity measurement as a result of variation between assessors in scoring of patients. However, all scores were determined by trained staff and each score was independently calculated by at least two health professionals; any discrepancies were discussed and resolved. Furthermore, PRISM has been validated as a predictive score for mortality in $\mathrm{MD} .{ }^{10}$ Whatever the limitations of PRISM (including other recent work which like ours also suggests that outcome in PICU patients admitted in the 1990s has improved so that PRISM now overestimates the mortality $\operatorname{risk}^{24}$ ), the consistent determination of the PRISM score throughout the course of this study has allowed an analysis of the trend in case fatality rate and shown a dramatic improvement in outcome.

Improvements in survival rates may be offset by a greater rate of serious morbidity in survivors. However, outpatient follow up performed six to eight weeks after hospital discharge did not show evidence for a greater risk of either amputation/skin grafting or neurological damage in 1996-97 compared with 1992-95.

Considerable changes in the management of patients with MD have occurred over the study period. While no single factor alone is likely to explain the reduction in mortality, several factors might have contributed to the improved outcome. In the past, few centres, including those with paediatric intensive care units, admitted more than a small number of patients with MD annually. Furthermore, patients were often considered too sick to transfer to a specialist centre and were treated in the accident \& emergency department, paediatric ward, or adult intensive care unit of the local district general hospital. Establishment of a mobile intensive care team allowed the centralisation of care of children with $\mathrm{MD}$ at a specialist clinical and research unit, which in turn enabled extensive experience in the management of MD to be developed; this may be the most important reason for the improved outcome. During 1997, every week there were on average two patients with MD admitted to the PICU.

The programme of education of clinicians in district hospitals has resulted in advice often being sought within minutes of the patient arriving in a local accident and emergency department. A telephone dialogue, established between the local physician and a paediatric intensivist with extensive experience in the disease, is likely to have influenced the initial resuscitation. An appreciation of the rapidity with which the disease can progress has enabled recommendations (on fluid resuscitation, earlier use of inotropes, and, in particular, early elective ventilation to facilitate redistribution of cardiac output ${ }^{19}$ ) to be made with confidence. As a result, patients who might previously have died in unresuscitated shock have survived until the arrival of the mobile intensive care team.

The ability to deliver a similar standard of paediatric intensive care, in a local general hospital's accident and emergency department or paediatric ward, to that available on a specialist unit by sending a mobile intensive care team of trained individuals (with appropriate equipment), has probably been another important factor in improved outcome through enabling the most severely ill children to survive the early hours of the disease. Patients were only transported back to St Mary's PICU once they had been fully resuscitated, stabilised, and monitored. The risk of interhospital transfer has been shown to be reduced by this approach. ${ }^{25}$

In addition to improved airway, breathing, and circulatory support of children with MD, an increased appreciation of the importance of early correction of electrolyte imbalance (including hypokalaemia, hypocalcaemia, hypomagnesaemia, hypoglycaemia, acidaemia, and hypophosphataemia) may also have improved outcome. Monitoring and correction of electrolyte imbalance and acid-base status are initially undertaken on an hourly basis. ${ }^{16} 1920$

As well as aggressive replacement of circulating volume with $4.5 \%$ human albumin solution, fresh frozen plasma, and blood, and the early use of inotropic agents in patients with persistent shock, there have been a number of changes in the support of multiorgan failure. Renal replacement therapy, utilising either peritoneal dialysis or haemofiltration, has been initiated earlier to prevent the development of electrolyte imbalance and fluid overload in patients with impaired renal function. Management of severe respiratory failure and acute respiratory distress syndrome may have been improved by use of newer means of mechanical ventilation, such as high frequency oscillatory ventilation, ${ }^{26}$ and also by inhaled nitric oxide.

While it is impossible to define the relative contributions to improved outcome of each of the many changes which have occurred in paediatric intensive care on this unit over the past few years, it is clear that current practice is producing a substantially lower case fatality rate than has been seen in previously published series of patients with severe meningococcal sepsis. ${ }^{3-8}$ The improvement in outcome over time necessitates critical evaluation of claims for beneficial effects of a number of experimental treatments such as ECMO, haemofiltration, and anticoagulant fibrinolytic agents, which have been made as a result of uncontrolled trials or using only historical controls. ${ }^{27}$

In conclusion, we have shown that a notable reduction in the case fatality rate for $\mathrm{MD}$ has been achieved. This may have been contributed to by increased expertise as a result of centralisation of cases at a specialist unit, 
greater interaction between the specialist centre and local hospitals to improve early management of patients prior to their transport to specialist centres, the use of a specialist retrieval service, and by a variety of improvements in the quality of intensive care. Application of this model elsewhere may produce similar improvement.

This work was supported by a programme grant from Meningitis Research Foundation. RB was a Wellcome fellow in epidemiology.

1 Jones D. Epidemiology of meningococcal disease in Europe and the USA. In: Cartwright K, ed. Meningococcal disease. New York: John Wiley \& Sons, 1995:147-57.

2 Achtman $M$. Global epidemiology of meningococcal disease. In: Cartwright K, ed. Meningococcal disease. New York: John Wiley \& Sons, 1995:159-75.

3 Havens PL, Garland JS, Brook MM, et al. Trends in mortality in children hospitalised with meningococcal infections, 1957 to 1987. Pediatr Infect Dis 7 1989:8:8-11.

4 Cartwright $\mathrm{K}$. Introduction and historical aspects. In: Cartwright K, ed. Meningococcal disease. New York: John Wiley \& Sons, $1995: 1-9$.

5 Duncan A. New therapies for severe meningococcal disease buncan A. New therapies for severe meningococc

6 Kirsch EA, Barton RP, Kitchen L, Giroir BP. PathophysiolKirsch EA, Barton RP, Kitchen L, Giroir BP. Pathophysiology, treatment and outcome of meningococcemia: a review

7 Derkx HHF, van den Hoek J, Redekop WK, et al. MeningoDerkx HHF, van den Hoek J, Redekop WK, et al. Meningococcal disease: a comparison of eight severity

8 Leclerc F, Hazelzet J, Jude B, et al. Protein C and S deficiency in severe infectious purpura of children: collaborative study of 40 cases. Intensive Care Med 1992;18: $1-4$.

9 Pollack MM, Ruttiman UE, Getson PR. Paediatric risk of mortality (PRISM) score. Crit Care Med 1988;16:1110-16.

10 Algren JT, Lal S, Cutliff SA, Richman B. Predictors of outcome in acute meningococcal infection in children. Crit Care Med 1993;21:447-52.

11 Goldman AP, Kerr SJ, Butt W, et al. Extracorporeal support for intractable cardiorespiratory failure due to meningofor intractable cardiorespiratory failure

12 Best C, Walsh J, Sinclair J, Beattie J. Early haemodiafiltration in meningococcal septicaemia. Lancet 1995; 347:202.
13 Zenz W, Muntean W, Gallisti S, et al. Recombinant tissue plasminogen activator treatment in two infants with fulminant meningococcemia. Paediatrics 1995;96:144-8.

14 Smith OP, White B, Vaughan D, et al. Use of protein-C concentrate, heparin, and haemodiafiltration in meningococcus-induced purpura fulminans. Lancet 1997; 350:1590-3.

15 Giroir BP, Quint PA, Barton P, et al. Preliminary evaluation of recombinant amino-terminal fragment of human bactericidal/permeability increasing protein in children with severe meningococcal sepsis. Lancet 1997;350:143943.

16 Nadel S, Levin M, Habibi P. Treatment of meningococcal disease in childhood. In: Cartwright K, ed. Meningococcal disease. New York: John Wiley \& Sons, 1995:207-43.

17 Pollack MM. Paediatric transport research: it is improving (finally). Crit Care Med 1994;22:1073-4.

18 Chief Medical Officer. Meningococcal septicaemia and meningitis. Circular to all medical practitioners in England and Wales, 1988

19 Pollard AJ, Britto J, Nadel S, et al. Emergency management of meningococcal disease. Arch Dis Child 1999:80:290-6.

20 Pathan N, Nadel S, Levin M. Pathophysiology and management of meningococcal septicaemia. $7 \mathrm{R}$ Coll Physicians Lond 2000;34 436-44.

21 Derkx B, Wittes J, McCLoskey R, and the European Pediatric Meningococcal Septic Shock Trial Study Group. Randomised, placebo-controlled trial of HA-1A, a human monoclonal antibody to endotoxin, in children with meningococcal septic shock. Clin Infect Dis 1999;28:770-7.

22 Levin M, Quint PA, Goldstein B, et al. Recombinant bactericidal/permeability-increasing protein (rBPI) as adjunctive treatment for children with severe meningococcal sepsis: a randomised trial. Lancet 2000;356:961-7.

3 Ramsay M, Kaczmarski E, Rush M, et al. Changing patterns of case ascertainment and trends in meningococcal disease in England and Wales. CDR Rev 1997;7:R49-54.

24 Tilford JM, Roberson PK, Lensing S, Fiser DH. Differences in paediatric ICU mortality risk over time. Crit Care Med 1998;26:1737-43

25 Britto J, Nadel S, Maconochie I, et al. Morbidity and severity of illness during interhospital transfer: impact of a specialised paediatric retrieval team. BMF 1995;311:836-9.

26 Paulson TE, Spear RM, Silva PD, et al. High-frequency pressure-control ventilation with high positive endexpiratory pressure in children with acute respiratory distress syndrome. F Pediatr 1996;129:566-73.

27 DeMunter C, Nadel S, Britto J, et al. ECMO for refractory cardiorespiratory failure due to meningococcal disease. Lancet 1997;349:1398.

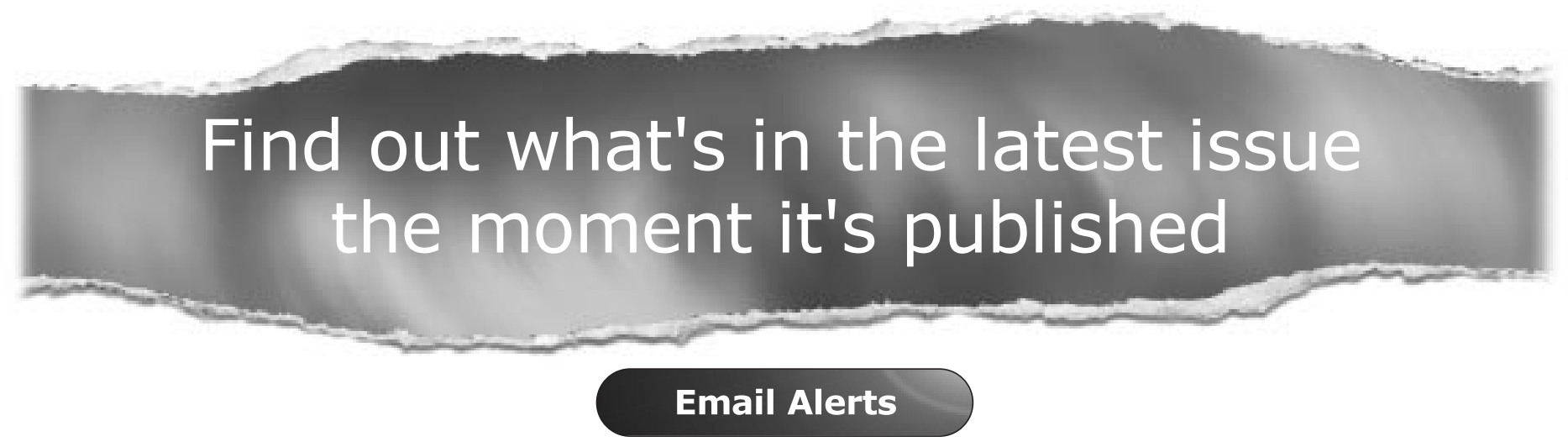

Sign up to receive the table of contents by email every month. You can select from three alerts: Table of Contents (full), TOC Awareness (notice only); Archives of Disease in Childhood related announcements.

www.archdischild.com 